\title{
Treatment outcome of high-dose image-guided intensity-modulated radiotherapy using intra-prostate fiducial markers for localized prostate cancer at a single institute in Japan
}

Ken Takeda ${ }^{1 *}$, Yoshihiro Takai ${ }^{2}$, Kakutaro Narazaki $^{3}$, Masatoshi Mitsuya $^{4}$, Rei Umezawa ${ }^{5}$, Noriyuki Kadoya ${ }^{5}$, Yukio Fujita ${ }^{5}$, Toshiyuki Sugawara ${ }^{5}$, Masaki Kubozono ${ }^{5}$, Eiji Shimizu ${ }^{5}$, Keiko Abe ${ }^{5}$, Yuko Shirata ${ }^{5}$, Yohjiro Ishikawa ${ }^{5}$, Takaya Yamamoto ${ }^{5}$, Maiko Kozumi ${ }^{5}$, Suguru Dobashi ${ }^{1}$, Haruo Matsushita ${ }^{5}$, Koichi Chida', Shigeto Ishidoya ${ }^{6}$, Yoichi Arai ${ }^{6}$, Keiichi Jingu ${ }^{5}$ and Shogo Yamada ${ }^{5}$

\begin{abstract}
Background: Several studies have confirmed the advantages of delivering high doses of external beam radiotherapy to achieve optimal tumor-control outcomes in patients with localized prostate cancer. We evaluated the medium-term treatment outcome after high-dose, image-guided intensity-modulated radiotherapy (IMRT) using intra-prostate fiducial markers for clinically localized prostate cancer.
\end{abstract}

Methods: In total, 141 patients with localized prostate cancer treated with image-guided IMRT (76 Gy in 13 patients and 80 Gy in 128 patients) between 2003 and 2008 were enrolled in this study. The patients were classified according to the National Comprehensive Cancer Network-defined risk groups. Thirty-six intermediate-risk patients and 105 high-risk patients were included. Androgen-deprivation therapy was performed in 124 patients (88\%) for a median of 11 months (range: 2-88 months). Prostate-specific antigen (PSA) relapse was defined according to the Phoenix-definition (i.e., an absolute nadir plus $2 \mathrm{ng} / \mathrm{ml}$ dated at the call). The 5-year actuarial PSA relapse-free survival, the 5-year distant metastasis-free survival, the 5-year cause-specific survival (CSS), the 5-year overall survival (OS) outcomes and the acute and late toxicities were analyzed. The toxicity data were scored according to the Common Terminology Criteria for Adverse Events, version 4.0. The median follow-up was 60 months.

Results: The 5-year PSA relapse-free survival rates were 100\% for the intermediate-risk patients and $82.2 \%$ for the high-risk patients; the 5-year actuarial distant metastasis-free survival rates were 100\% and 95\% for the intermediateand high-risk patients, respectively; the 5-year CSS rates were 100\% for both patient subsets; and the 5-year OS rates were $100 \%$ and $91.7 \%$ for the intermediate- and high-risk patients, respectively. The Gleason score ( $<8$ vs. $\geq 8$ ) was significant for the 5-year PSA relapse-free survival on multivariate analysis $(p=0.044)$. There was no grade 3 or 4 acute toxicity. The incidence of grade 2 acute gastrointestinal (GI) and genitourinary (GU) toxicities were $1.4 \%$ and $8.5 \%$, respectively. The 5-year actuarial likelihood of late grade $2-3 \mathrm{Gl}$ and GU toxicities were $6 \%$ and $6.3 \%$, respectively. No grade 4 Gl or GU late toxicity was observed.

Conclusions: These medium-term results demonstrate a good tolerance of high-dose image-guided IMRT. However, further follow-up is needed to confirm the long-term treatment outcomes.

Keywords: Image-guided radiotherapy, Prostate cancer, Biochemical control, Toxicity

\footnotetext{
* Correspondence: ktakeda-rad@umin.net

'Department of Radiological Technology, School of Health Sciences, Faculty

of medicine, Tohoku University, Sendai, Japan

Full list of author information is available at the end of the article
} 


\section{Background}

Recently, several studies have confirmed the advantages of delivering high doses of external beam radiotherapy (EBRT) to achieve optimal tumor-control outcomes in patients with localized prostate cancer. It is now clear that conventional EBRT doses in the range of 70 Gy are not sufficient to eradicate local prostate disease [1,2]. In addition, a higher EBRT dose requires greater accuracy and precision. Thus, various position verification methods, including image-guided radiotherapy, have been developed, and their effectiveness has been reported [3]. However, there are few publications regarding the treatment outcome after completion of the delivery of high-doses with image-guided intensitymodulated radiotherapy (IMRT) using daily intraprostatic fiducial marker-based position verification for localized prostate cancer [4].

We investigated the medium-term treatment outcomes, including the 5-year prostate-specific antigen (PSA) relapse-free survival rate, the 5-year distant metastasis-free survival rate, the 5-year cause-specific survival (CSS) rate, the 5-year overall survival (OS) rate and the toxicity outcomes, after high-dose image-guided IMRT using daily intra-prostatic fiducial markers in patients with clinical localized prostate cancer.

\section{Methods}

Between 2003 and 2008, 150 patients with localized prostate cancer were treated with image- guided IMRT at Tohoku University Hospital. Among these patients, 141 patients who satisfied the eligibility criteria were included in the current study and retrospectively analyzed.

\section{Eligibility criteria}

The eligible patients had a biopsy-confirmed adenocarcinoma of the prostate with the clinical stage T1-3N0M0 and were classified in the National Comprehensive Cancer Network (NCCN)-defined (www.nccn.com) intermediateor high-risk groups. Each patient received magnetic resonance imaging before the initial treatment to exclude lymph node metastasis and for the staging procedure. Patients with T2b or T2c clinical stage tumors, a Gleason score (GS) of 7, or a pretreatment PSA level between 10 and $20 \mathrm{ng} / \mathrm{mL}$ were classified as intermediate-risk disease. Patients who had tumors with a T3a clinical stage or higher, a $G S \geq 8$, or a pretreatment PSA level $>20 \mathrm{ng} / \mathrm{mL}$ were classified as having high-risk disease.

\section{Exclusion criteria}

NCCN-defined low-risk patients with a T1-2a clinical stage tumor, a GS $<7$, a pretreatment PSA level $<10 \mathrm{ng} /$ $\mathrm{mL}$, and N1 disease were not enrolled in this study. Patients with a T4 clinical stage tumor, the presence of metastasis, other concurrent invasive cancers, or active collagen disease were also not included. Additionally, patients with salvage intent were not enrolled, including patients with a biochemical relapse following a prior prostatectomy, prior pelvic radiotherapy, and hormonal therapy. Patients with a follow-up period within 1 year were also not registered in this analysis.

A total of 105 patients with high-risk localized prostate cancer and 36 patients with intermediate-risk localized prostate cancer received IMRT up to a prescribed dose of $76 \mathrm{~Gy}$ or $80 \mathrm{~Gy}$ and were investigated. The patient characteristics are listed in Table 1. Pelvic lymph node dissection (PLND) was performed in 45 patients (32\%) to rule out metastatic disease.

All eligible patients provided written informed consent before treatment. The institutional research ethics board approved this study.

\section{Radiotherapy}

The detailed techniques for IMRT treatment planning and delivery have been previously reported [5]. Briefly, each patient was implanted with three gold fiducial markers in the prostate gland before the treatment-planning computerized tomography (CT) scan was acquired. All patients were immobilized in the supine position with a vacuum bag system for their entire body. Since January

Table 1 Patient characteristics

\begin{tabular}{|c|c|c|c|c|}
\hline \multicolumn{3}{|l|}{ Age (y) } & \multirow{3}{*}{$\begin{array}{l}\frac{\text { Median (range) }}{\mathbf{N}} \\
34\end{array}$} & \multirow{3}{*}{$\begin{array}{l}71(50-83) \\
\% \\
24\end{array}$} \\
\hline \multirow{4}{*}{ T stage } & & & & \\
\hline & $\mathrm{T} 1$ & & & \\
\hline & $\mathrm{T} 2$ & & 40 & 28 \\
\hline & $\mathrm{T} 3$ & & 67 & 48 \\
\hline \multirow[t]{2}{*}{ Gleason score } & $<8$ & & 73 & 52 \\
\hline & $8-10$ & & 68 & 48 \\
\hline \multirow[t]{2}{*}{ Initial PSA } & $\leq 20$ & & 93 & 66 \\
\hline & $>20$ & & 48 & 34 \\
\hline \multirow[t]{2}{*}{ NCCN riskGroup } & $\mathbb{R}$ & & 36 & 26 \\
\hline & $\mathrm{HR}$ & & 105 & 74 \\
\hline \multirow[t]{6}{*}{ ADT } & & Yes & 124 & 88 \\
\hline & & IR & 24 & 17 \\
\hline & & $H R$ & 100 & 71 \\
\hline & & STADT & 27 & 22 \\
\hline & & LTADT & 97 & 78 \\
\hline & & No & 17 & 12 \\
\hline Diabetes & & & 23 & 16 \\
\hline Hypertension & & & 50 & 35 \\
\hline Hemorrhoid & & & 37 & 26 \\
\hline
\end{tabular}

Abbreviations: PSA, prostate-specific antigen; NCCN, national comprehensive cancer network; IR, intermediate risk; $H R$, high risk; $A D T$, androgen-deprivation therapy; STADT, short-term ADT; LTADT, long-term ADT. 
2004, we have temporarily used a urethral catheter for identification of the urethra on radiotherapy-planning CT images and for contouring the urethra after image acquisition. The CT scans were then performed at a 2.5$\mathrm{mm}$ slice thickness. In the IMRT planning, Eclipse (release 6.5; Varian medical Systems, Palo Alto, CA, USA) was used for dose calculations. The clinical target volume (CTV) included the prostate and seminal vesicles. To adequately encompass the extent of tumor invasion in the seminal vesicles, the CTV involved the base of the seminal vesicles in T1-3a patients and more distal to entire portion of the seminal vesicles in T3b patients, respectively. Based on our previous study [6], the CTV was expanded in three dimensions with a $0.5-\mathrm{cm}$ margin to obtain the planning target volume (PTV) with the exception of the prostate-rectum interface, where a 0.3$\mathrm{cm}$ margin was adopted to decrease rectal involvement. A portion of the rectal wall located at the level of the PTV and $0.5 \mathrm{~cm}$ outside of the PTV on the CT images was contoured. The rectum, bladder, bowel, and femur were contoured as critical normal tissue structures. The rectal wall was defined with a 2-mm internal wall extraction. The bladder was entirely contoured, and a 5-mm inner wall defined the bladder wall volume.

IMRT was delivered using 15MV photons generated by a Clinac 23 EX linear accelerator (Varian medical Systems, Palo Alto, CA). The standard 5-8 coplanar beams were used. The prescribed dose used to cover $95 \%$ of the target volume $\left(\mathrm{D}_{95}\right)$ was 76 Gy in 13 patients and 80 Gy in 128 patients. A total dose of 76 Gy in 2-Gy daily fractions was delivered to 13 patients who had other prior severe diseases, including diabetes or cardiovascular disorders. The maximum dose heterogeneity allowable in the PTV was $10 \%$. Each treatment plan was optimized to ensure the following conditions: no more than $65 \%$ of the rectal and urinary bladder wall received $>35$ Gy $\left(\mathrm{V}_{35} \leq 65 \%\right)$; no more than $45 \%$ of the rectal and urinary bladder wall received $>55$ Gy $\left(\mathrm{V}_{55} \leq 45 \%\right)$; no more than $25 \%$ of the rectal and urinary bladder wall received $>75$ Gy $\left(\mathrm{V}_{75} \leq 25 \%\right)$; and the urethral, rectal, and bladder walls received no more than $80 \mathrm{~Gy}$. In the overlap region between the PTV and these critical organs, the constraint was set to $95 \%$ of the prescription dose for the rectum and $95 \%$ for the urethra. The latter dose constraint for the urethra has been applied to 108 patients since January 2004.

In addition, prior to the acquisition of the treatment planning CT images and 30 minutes before the daily IMRT, each patient urinated to ensure the bladder was in the same state. In addition, the patients emptied their bowels just before the daily IMRT. For every treatment fraction, the patients were initially prepared using laser marks on their skin, and they were then repositioned using the Varian On-Board Imager based on the positions of the three intra-prostatic fiducial markers, and the precision-of-position verification was within $1 \mathrm{~mm}$. We did not find any instances of fiducial migration during treatment. All patients were treated to their prescribed dose in daily 2.0 Gy fractions. The imaging dose was not accounted for in the treatment plans.

\section{Hormonal therapy}

Androgen-deprivation therapy (ADT) was used at the discretion of the treating physician. ADT primarily consisted of an oral anti-androgen and luteinizing hormonereleasing hormone agonist administered as depot injections. In 124 patients receiving $\mathrm{ADT}$, the median $\mathrm{ADT}$ duration was 11 months (range: 2-88 months). Among these patients, 44 (35\%) received only neoadjuvant ADT, and the other $80(65 \%)$ were treated with neoadjuvant ADT, concurrent and adjuvant ADT. Table 1 shows the details of the ADT duration. ADT was classified as short-term (STADT) if it was administered for $\leq 6$ months and long-term (LTADT) if it was administered for $>6$ months. The median duration of ADT in the 24 intermediate-risk and 100 high-risk patients was 5 months (range: 4-32 months) and 12 months (range: 2-88 months), respectively. The details of STADT and LTADT are as follows: for the 24 intermediate-risk patients, there were 10 STADT and 14 LTADT, and for the 100 high-risk patients, there were 17 STADT and 83 LTADT. The median duration between the initiation of ADT and the start of IMRT was 8 months (range: 136 months).

\section{After treatment follow-up evaluation}

Follow-up evaluations after the completion of treatment were performed at 3- to 6-month intervals for 5 years and every 6 months thereafter. The median follow-up was 66 months (range: 17-111 months).

Freedom from biochemical relapse was analyzed using the Phoenix consensus definition (i.e., an absolute nadir PSA level plus $2 \mathrm{ng} / \mathrm{mL}$ more than the recorded level) $[7,8]$. For CSS analysis, patients with documentation of biochemical or metastatic relapsed disease who subsequently died were scored as deaths from localized prostate cancer.

Acute and late toxicity data were scored according to the National Cancer Institute-designated Common Terminology Criteria for Adverse Events Version 4.0.

\section{Statistical analyses}

The distributions of the 5-year PSA relapse-free survival were calculated using Kaplan-Meier curves for biochemical control using the one failure definition. The 5-year actuarial distant metastasis-free survival, CSS, and OS rates were also evaluated by Kaplan-Meier curves. Univariate analyses (UA) and multivariate analyses (MA) were 
performed to determine the related PSA relapse-free survival predictors (i.e., NCCN risk stratification, GS, ADT use and duration, STADT, LTADT, pretreatment PSA, and PLND) and the late gastrointestinal (GI) and genitourinary (GU) side effects $\geq$ grade 2 (i.e., age, ADT use, duration between ADT initiation and the start of IMRT, the presence of diabetes, hypertension, hemorrhoids, acute grade $2 \mathrm{GI}$ and GU toxicities, and prescribed RT dose). MA was performed using a Cox regression model. Statistical analyses were performed using the Statistical Package for Social Sciences for Windows, version 20. A $p$-value $<0.05$ (two-sided) was considered to be statistically significant in all tests.

\section{Results}

\section{Biochemical tumor-control rate}

The 5-year actuarial PSA relapse-free survival outcomes for the intermediate- and high-risk groups were 100 and $82.2 \%$, respectively [Figure 1], and the NCCN risk classification, GS, and pretreatment PSA were significant in UA, whereas only the GS was statistically significant variable in MA [Table 2].

\section{Distant metastasis-free survival, cause-specific survival and overall survival rates}

Distant metastases developed in four (2.8\%) patients. The 5-year actuarial distant metastasis-free survival rates for the intermediate- and high-risk groups were 100 and
Table 2 Statistical analyses of predictors for the 5-year PSA relapse-free survival, $p$ values

\begin{tabular}{lll}
\hline & UA & MA \\
\hline NCCN risk (IR vs. HR) & $0.01^{*}$ & 0.96 \\
GS (8-10 vs. <8) & $<0.001^{*}$ & $0.044^{*}$ \\
ADT (yes vs. no) & 0.1 & 0.967 \\
ADT duration (continuous) & 0.452 & 0.549 \\
STADT vs. LTADT & 0.477 & 0.513 \\
Pretreatment PSA (<20 vs. >20) & $0.046^{*}$ & 0.29 \\
PLND (yes vs. no) & 0.316 & 0.454
\end{tabular}

Abbreviations: UA, univariate analysis; MA, multivariate analysis; ${ }^{\text {, }}$ statistical significance; PLND, pelvic lymph node dissection; other abbreviations as in Table 1.

95\%, respectively [Figure 2]. The 5-year CSS rates for the intermediate- and high-risk patients were both $100 \%$. The 5-year OS rates for the intermediate- and high-risk patients were 100 and $91.7 \%$, respectively.

\section{Toxicity}

Grades 1 and 2 GI acute toxicity were identified in 29 (20\%) and 2 (1.4\%) patients, respectively. Grades 1 and 2 acute GU toxicities occurred in $84(60 \%)$ and $12(8.5 \%)$ patients, respectively. There was no acute GI and GU toxicity grade 3 or higher.

Late grade 2 or 3 GI toxicities developed in eight patients $(5.7 \%)$ at a median of 18 months after image-

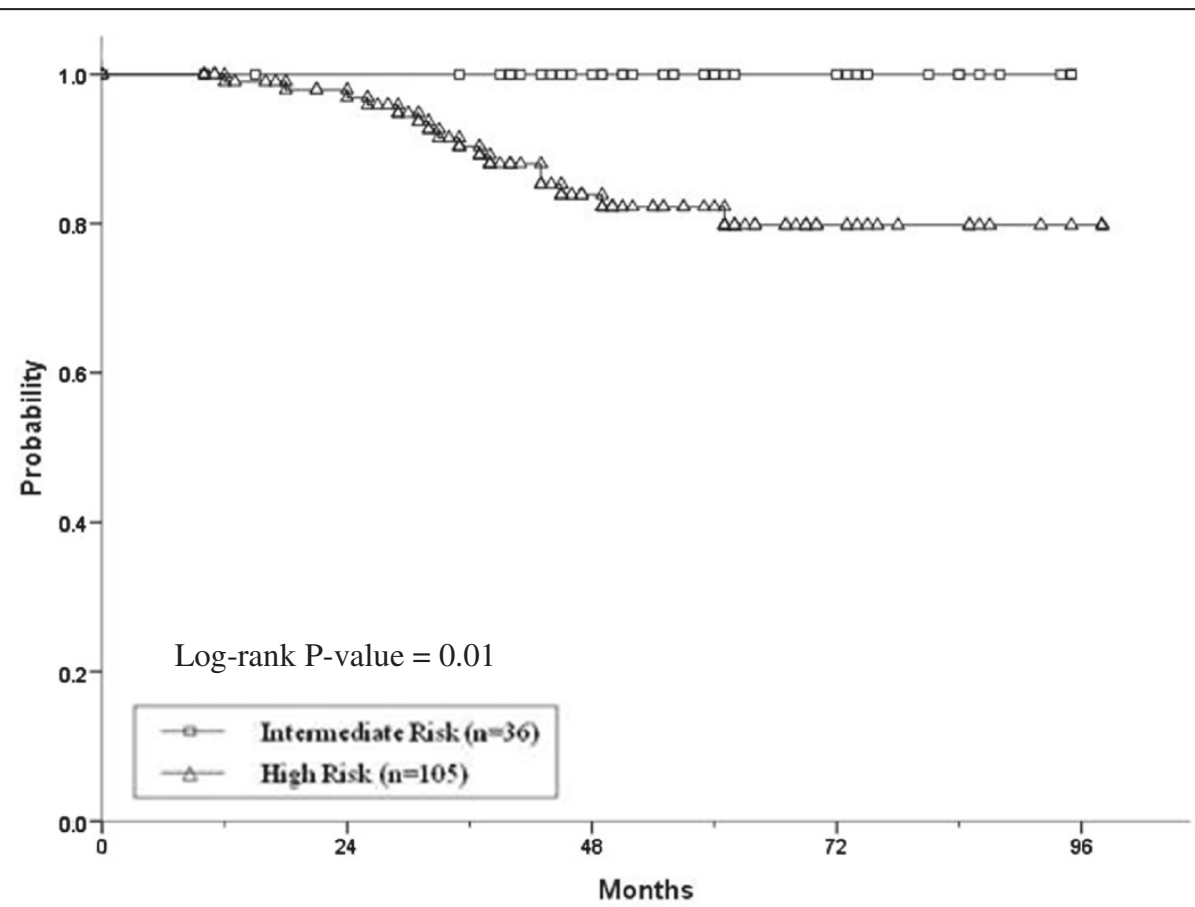

Figure 1 Phoenix consensus definition PSA relapse-free survival by NCCN risk group. Abbreviations: PSA, prostate-specific antigen; NCCN, national comprehensive cancer network. 


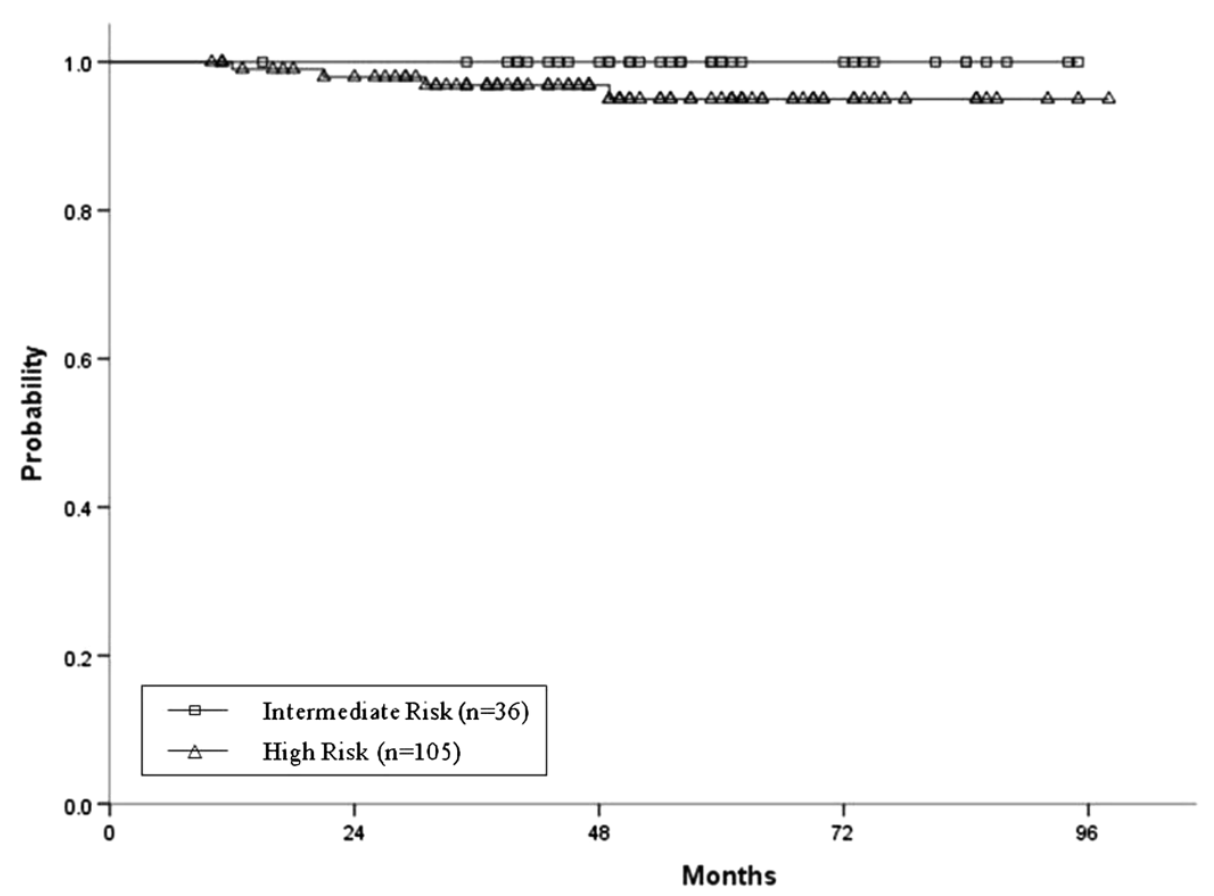

Figure 2 The Kaplan-Meier curve illustrates the actuarial probability of achieving distant metastasis-free survival.

guided IMRT. There was rectal hemorrhage in seven patients and proctitis in one patient. Rectal hemorrhage as a grade 3 late GI toxicity occurred in two patients who were treated with several transfusions and a laser cauterization procedure. No grade 4 or greater GI complications were observed. The 5 -year actuarial likelihood of late grade 2 or 3 GI toxicities was 6\% [Figure 3]. In UA, the group without ADT was related to grade 2 or 3 late GI toxicities, whereas that relationship was not significant in MA [Table 3]. In addition, the duration

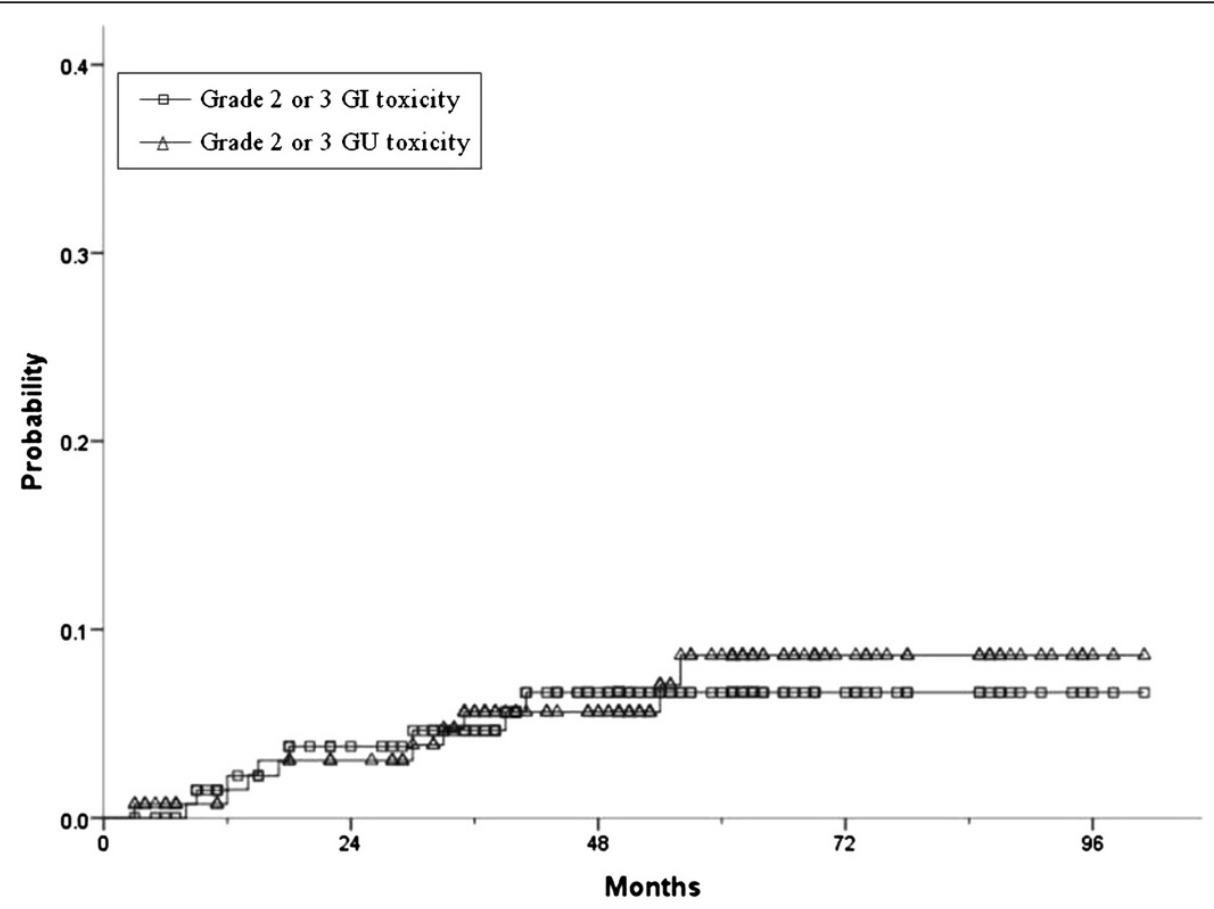

Figure 3 Kaplan-Meier actuarial probability of late grade 2 or $3 \mathrm{GI}$ and GU toxicities. Abbreviations: Gl, Gastrointestinal; GU, Genitourinary. 
Table 3 Statistical analyses of predictors for late toxicity, $p$ values

\begin{tabular}{|c|c|c|c|c|}
\hline & \multicolumn{2}{|c|}{$\begin{array}{l}\text { Late grade } 2 \text { or } \\
3 \mathrm{Gl} \text { toxicity }\end{array}$} & \multicolumn{2}{|c|}{$\begin{array}{l}\text { Late grade } 2 \text { or } \\
3 \mathrm{GU} \text { toxicity }\end{array}$} \\
\hline & $\overline{U A}$ & MA & UA & MA \\
\hline Age (>71) & 0.414 & 0.42 & 0.078 & $0.039^{*}$ \\
\hline ADT & $0.025^{*}$ & 0.401 & 0.889 & 0.314 \\
\hline $\begin{array}{l}\text { Duration between ADT } \\
\text { initiation and the start } \\
\text { of IMRT }\end{array}$ & 0.088 & 0.619 & 0.522 & 0.312 \\
\hline Diabetes & 0.526 & 0.877 & 0.66 & 0.511 \\
\hline Hypertension & 0.396 & 0.474 & 0.38 & 0.732 \\
\hline Hemorrhoid & 0.952 & 0.853 & NA & NA \\
\hline Acute grade $2 \mathrm{Gl}$ toxicity & 0.743 & 0.992 & NA & NA \\
\hline Acute grade $2 \mathrm{GU}$ toxicity & NA & NA & $<0.001^{*}$ & $0.001^{*}$ \\
\hline 76 Gy vs. 80 Gy & 0.77 & 0.716 & 0.291 & 0.987 \\
\hline
\end{tabular}

Abbreviations: $\mathrm{Gl}$, gastrointestinal; GU, genitourinary; NA, not applicable; other abbreviations as in Tables 1-2.

between ADT initiation and the start of IMRT was also not statistically significant for late GI toxicities grade 2 or 3 [Table 3].

Late grade 2 or $3 \mathrm{GU}$ toxicities developed in nine patients $(6.4 \%)$ at a median of 30 months after imageguided IMRT. There was urinary retention in four patients, urinary frequency in two patients, urinary tract obstruction in one patient, urgency in one patient and non-infective cystitis in one patient. No grade 4 or greater GU toxicity was observed. The 5-year actuarial likelihood of late grade 2 or $3 \mathrm{GU}$ toxicities was $6.3 \%$ [Figure 3]. In UA and MA, acute grade $2 \mathrm{GU}$ toxicity was predictive of late grade 2 or $3 \mathrm{GU}$ toxicities [Table 3]. Although age $(>71)$ was not significant in UA, it was predictive in the MA [Table 3].

\section{Discussion}

Our results show that high-dose image-guided IMRT can be safely performed and is well tolerated. This study also demonstrated relatively few biochemical failures after image-guided IMRT in patients with intermediate- and high-risk localized prostate cancer as a medium-term period assessment [Table 4]. Nevertheless, it is difficult to tell whether this was a function of high-dose image-guided IMRT, heterogeneity within this series of patients, different hormonal therapies, or any combination of these factors.

There is clear support for dose escalation in prostate cancer radiotherapy. Several studies have confirmed that 74- to $81-$ Gy doses provided a $15-20 \%$ improvement in biochemical control compared with conventional doses of $<70$ Gy [9-12]. According to previous studies, biochemical tumor-control rate improvement will lead to better distant metastasis-free survival and CSS [13-15]. The current distant metastasis-free survival and CSS rates were at least equivalent to other reports, although direct comparison with other publications is difficult due to differences in the follow-up periods $[1,2,12]$.

Table 4 Literature review

\begin{tabular}{|c|c|c|c|c|c|c|}
\hline & RT dose (Gy) & & ADT rate (\%) & ADT duration (M) & PSA relaps & ial (\%) \\
\hline \multirow[t]{3}{*}{ Alicikus et al. [1] } & 81 & $\mathrm{LR}$ & 54 (total) & 3 & PD: 10-year & 81 \\
\hline & & $\mathbb{R}$ & & & & 78 \\
\hline & & $\mathrm{HR}$ & & & & 62 \\
\hline \multirow[t]{3}{*}{ Martin et al. [4] } & 79.8 & $L R$ & 13.6 & not mentioned & PD: 5-year & 88.4 \\
\hline & & $\mathbb{I R}$ & 11.0 & & & 76.5 \\
\hline & & $\mathrm{HR}$ & 45.9 & & & 77.9 \\
\hline \multirow[t]{6}{*}{ Zelefsky et al. [12] } & 81 & $L R$ & 33.5 & 3 & AD: 8-year & 85 \\
\hline & & $\mathbb{R}$ & 52 & & & 76 \\
\hline & & $\mathrm{HR}$ & 92 & & & 72 \\
\hline & & $L R$ & 33.5 & & PD: 8-year & 89 \\
\hline & & $\mathbb{R}$ & 52 & & & 78 \\
\hline & & $\mathrm{HR}$ & 92 & & & 67 \\
\hline \multirow[t]{3}{*}{ Cahlon, et al. [16] } & 86.4 & LR & 66 (total) & 3 or 9 & PD: 5-year & 98 \\
\hline & & $\mathbb{R}$ & & & & 85 \\
\hline & & $\mathrm{HR}$ & & & & 70 \\
\hline \multirow[t]{2}{*}{ Current study } & 80 or 76 & $\mathbb{I R}$ & 67 & 5 (median) (range: 4-32) & PD: 5-year & 100 \\
\hline & & $H R$ & 95 & 12 (median) (range: 2-88) & & 82.2 \\
\hline
\end{tabular}


In addition to high-dose image-guided IMRT, a relatively high rate and long duration of ADT administration may contribute to the current biochemical tumor-control outcome, although the effect was not statistically significant [Tables 2 and 4] [1,12,16,17]. To our knowledge, few publications have reported high-dose EBRT with a high rate and long ADT administration duration that was similar to this study. Moreover, according to D'Amico et al. and Bolla et al., the radiotherapy survival benefit may be improved by adding ADT for high- and intermediate-risk localized prostate cancer $[17,18]$. Furthermore, a metaanalysis of Radiation Therapy Oncology Group prostate cancer trials demonstrated that STADT appeared to improve the 8-year disease-specific survival for intermediaterisk patients, whereas LTADT improved the 8-year overall survival for high-risk patients [19]. According to Alicikus et al., the lack of a benefit from ADT in their high-risk patients may have been caused by the relatively short course of only 5-6 months of ADT in their study [1]. They advocated the use of a longer ADT course, particularly for high-risk patients.

The incidence of late grade $\geq 2$ GI and GU toxicity following high-dose radiotherapy in recent studies ranged from 3.7 to $22 \%$ and from 8.6 to $35 \%$, respectively $[1,4,16,20]$. In this report, the likelihood of developing late grade $\geq 2 \mathrm{GI}$ and GU toxicities was 6 and $6.3 \%$, respectively, at 5 years. One reason that may explain our late GI toxicity incidence may be that we did not confirm the actual position of the rectum during the entire IMRT period, although every patient was prepared before each treatment with minimized bowel contents, including gas and stool, which was similar to the acquisition of treatment-planning CT. Although Smeenk et al. reported some procedures for reducing the physiological bowel effects, those techniques were not used in this study [21].

The relationship between ADT administration and late grade $2-3$ GI toxicities was not statistically significant in MA, whereas there appeared to be a relationship in UA [Table 3]. In addition, the duration between ADT initiation and the start of IMRT was not correlated with late GI grade 2 or 3 toxicities [Table 3]. Although there may be some mechanisms that explain why patients with ADT administration demonstrated a low tendency for late grade 2-3 GI toxicities in UA, it is difficult to clarify. This topic is controversial. Several authors reported that ADT administration increases late GI toxicity $[20,22]$. In contrast, other studies have indicated lower GI and GU toxicity rates when ADT was added to EBRT for localized prostate cancer [23,24]. Longer follow-up and further investigation are needed.

The incidence of late grade 2-3 GU toxicities appeared to be lower than that previously reported $[1,4,16,20]$. Several reasons may exist. One reason might be that we set the urethral dose constraints to reduce the maximal urethral dose to $<80 \mathrm{~Gy}$ in patients treated with 80 Gy since January 2004. Zelefsky et al. indicated more severe late urinary toxicity following high-dose IMRT delivering 81.0 Gy or 86.4 Gy [25]. According to these authors, the 3-year actuarial likelihood of late grade $\geq 2$ urinary toxicities was $15 \%$. Therefore, we began setting the urethral dose constraints in 2004. Another reason might be that our image-guided IMRT had a precision-of-position verification within $1 \mathrm{~mm}$. However, further follow-up and examination is necessary to evaluate late GU toxicity and related factors.

In this series, acute grade $2 \mathrm{GU}$ toxicity was statistically related to late grade 2-3 GU toxicity in the MA [Table 3]. Alicikus et al. also demonstrated that acute grade $\geq 2$ GU toxicity was predictive for late grade $\geq 2$ GU toxicity [1]. According to Bolla et al. and Peeters et al., an increase in late GU toxicity was associated with LTADT in addition to RT $[17,22]$. In contrast, similar to Alicikus et al., the use and duration of ADT was not related to the late grade 2-3 GU toxicity in this study [1] [Table 3].

Grade 2 acute GI and GU symptoms developed in two $(1.4 \%)$ and 12 patients (8.5\%), respectively. There was no acute GI or GU toxicity of grade 3 or higher. Similar to Alicikus et al., the acute GI toxicity was minimal [1]. However, the current incidence of acute GU symptoms was higher than that in their study [1]. Alicikus et al. reported a grade 2 acute GU toxicity frequency of $3 \%$. Nevertheless, other studies have indicated higher occurrences of grade 2 acute GU toxicity than our study, ranging from 22 to 47\% [3,4,16]. Accordingly, our results, with respect to acute and late GI and GU toxicities, appear to be favorable.

Several limitations exist in this study. First, this study had no randomization; therefore, it had an inherent potential for selection bias. Additionally, our sample size was more limited than some other studies $[1,4,12,16]$. Second, this analysis included a small number of patients who were followed for $<5$ years, which did not allow an adequate period to evaluate clinical failures or side effects. The small number of biochemical failures could be a function of the short follow-up. Third, we unfortunately did not estimate sexual function before and after treatment in this analysis, whereas several authors have reported a relationship between $\mathrm{ADT}$ use and the development of erectile dysfunction [1,26,27]. However, we reported the health-related quality of life in smaller numbers of patients treated with image-guided IMRT [28], and further investigation is in progress.

\section{Conclusions}

We report the medium-term treatment outcomes of highdose image-guided IMRT for patients with localized 
prostate cancer. The current treatment is well tolerated and appears to provide valuable biochemical tumor control. Nevertheless, a relatively high rate and long duration of ADT administration may have also potentially contributed to the outcome. Further investigation is needed to optimize integration between dose-escalated radiotherapy and adequate ADT. Additionally, longer follow-up is essential to evaluate the long-term treatment outcomes.

\section{Abbreviations}

EBRT: External Beam Radiation Therapy; IMRT: Intensity-Modulated Radiation Therapy; PSA: Prostate-Specific Antigen; CSS: Cause-Specific Survival; OS: Overall Survival; NCCN: National Comprehensive Cancer Network; GS: Gleason Score; PLND: Pelvic Lymph Node Dissection; CT: Computerized Tomography; CTV: Clinical Target Volume; PTV: Planning Target Volume; ADT: Androgen-Deprivation Therapy; STADT: Short-Term ADT; LTADT: LongTerm ADT; UA: Univariate Analysis; MA: Multivariate Analysis; Gl: Gastrointestinal; GU: Genitourinary.

\section{Competing interests}

The authors declare that they have no competing interests.

\section{Authors contributions}

Study design: YT, KN, MM, SI, YA. Patient contribution, manuscript review: KT, YT, KN, MM, RU, NK, YF, TS, MK, ES, KA, YS, YI, TY, MK, SD, HM, KC, SI, YA, KJ, SY. Data collection: KT, YT, KN, RU, TS, MK, ES, KA, YS, YI, TY, MK, HM, SI, YA. Data analysis, manuscript preparation: KT. All authors reviewed and approved the final manuscript.

\section{Author details}

${ }^{1}$ Department of Radiological Technology, School of Health Sciences, Faculty of medicine, Tohoku University, Sendai, Japan. ${ }^{2}$ Department of Radiology and Radiation Oncology, Hirosaki University School of medicine, Hirosaki, Japan. ${ }^{3}$ Department of Radiation Oncology, Sendai medical Center, Sendai, Japan. ${ }^{4}$ Sendai Radiation Oncology and Imaging Clinic, Sendai, Japan. ${ }^{5}$ Department of Radiation Oncology, Tohoku University Hospital, Sendai, Japan. ${ }^{6}$ Department of Urology, Tohoku University Hospital, Sendai, Japan.

Received: 31 March 2012 Accepted: 2 July 2012

Published: 6 July 2012

\section{References}

1. Alicikus ZA, Yamada Y, Zhang Z, Pei X, Hunt M, Kollmeier M, Cox B, Zelefsky MJ: Ten-year outcomes of high-dose, intensity-modulated radiotherapy for localized prostate cancer. Cancer 2011, 117(7):1429-1437.

2. Beckendorf V, Guerif S, Le Prise E, Cosset JM, Bougnoux A, Chauvet B, Salem N, Chapet O, Bourdain S, Bachaud JM, Maingon P, Hannoun-Levi JM, Malissard L, Simon JM, Pommier P, Hay M, Dubray B, Lagrange JL, Luporsi E, Bey P: 70 Gy versus $80 \mathrm{~Gy}$ in localized prostate cancer: 5 -year results of GETUG 06 randomized trial. Int J Radiat Oncol Biol Phys 2011, 80(4):1056-1063.

3. Lips IM, Dehnad H, van Gils CH, Kruger AEB, van der Heide UA, van Vulpen M: High-dose intensity-modulated radiotherapy for prostate cancer using daily fiducial marker-based position verification: acute and late toxicity in 331 patients. Radiat Oncol 2008, 3:15.

4. Martin JM, Bayley A, Bristow R, Chung P, Gospodarowicz M, Menard C, Milosevic M, Rosewall T, Warde PR, Catton CN: Image guided dose escalated prostate radiotherapy: still room to improve. Radiat Oncol 2009, 4:50.

5. Takeda K, Ogawa Y, Ariga H, Koto M, Sakayauchi T, Fujimoto K, Narazaki K, Mitsuya M, Takai Y, Yamada S: Clinical correlations between treatment with anticoagulants/antiaggregants and late rectal toxicity after radiotherapy for prostate cancer. Anticancer Res 2009, 29(5):1831-1834.

6. Britton KR, Takai Y, Mitsuya M, Nemoto K, Ogawa Y, Yamada S: Evaluation of inter- and intrafraction organ motion during intensity-modulated radiation therapy (IMRT) for localized prostate cancer measured by a newly developed on-board image-guided system. Radiat med 2005, 23(1):14-24.

7. Thames H, Kuban D, Levy L, Horwitz EM, Kupelian P, Martinez A, Michalski J, Pisansky T, Sandler H, Shipley W, Zelefsky M, Zietman A:
Comparison of alternative biochemical failure definitions based on clinical outcome in 4839 prostate cancer patients treated by external beam radiotherapy between 1986 and 1995. Int J Radiat Oncol Biol Phys 2003, 57(4):929-943.

8. Roach M 3rd, Hanks G, Thames H, Schellhammer P, Shipley WU, Sokol GH, Sandler H: Defining biochemical failure following radiotherapy with or without hormonal therapy in men with clinically localized prostate cancer: recommendations of the RTOG-ASTRO Phoenix Consensus Conference. Int J Radiat Oncol Biol Phys 2006, 65(4):965-974.

9. Zelefsky MJ, Leibel SA, Gaudin PB, Kutcher GJ, Fleshner NE, Venkatramen ES, Reuter VE, Fair WR, Ling CC, Fuks Z: Dose escalation with threedimensional conformal radiation therapy affects the outcome in prostate cancer. Int J Radiat Oncol Biol Phys 1998, 41(3):491-500.

10. Heemsbergen WD, Peeters ST, Koper PC, Hoogeman MS, Lebesque JV: Acute and late gastrointestinal toxicity after radiotherapy in prostate cancer patients: consequential late damage. Int J Radiat Oncol Biol Phys 2006, 66(1):3-10.

11. Pollack A, Zagars GK, Starkschall G, Antolak JA, Lee JJ, Huang E, von Eschenbach AC, Kuban DA, Rosen I: Prostate cancer radiation dose response: results of them. D. Anderson phase III randomized trial. Int J Radiat Oncol Biol Phys 2002, 53(5):1097-1105.

12. Zelefsky MJ, Chan H, Hunt M, Yamada Y, Shippy AM, Amols H: Long-term outcome of high dose intensity modulated radiation therapy for patients with clinically localized prostate cancer. J Urol 2006, 176(4 Pt 1):1415-1419.

13. Jacob R, Hanlon AL, Horwitz EM, Movsas B, Uzzo RG, Pollack A: The relationship of increasing radiotherapy dose to reduced distant metastases and mortality in men with prostate cancer. Cancer 2004, 100(3):538-543.

14. Kupelian PA, Ciezki J, Reddy CA, Klein EA, Mahadevan A: Effect of increasing radiation doses on local and distant failures in patients with localized prostate cancer. Int J Radiat Oncol Biol Phys 2008, 71(1):16-22.

15. Zelefsky MJ, Yamada Y, Fuks Z, Zhang Z, Hunt M, Cahlon O, Park J, Shippy A: Long-term results of conformal radiotherapy for prostate cancer: impact of dose escalation on biochemical tumor control and distant metastases-free survival outcomes. Int J Radiat Oncol Biol Phys 2008 71(4):1028-1033.

16. Cahlon O, Zelefskym J, Shippy A, Chan H, Fuks Z, Yamada Y, Hunt M Greenstein S, Amols H: Ultra-high dose (86.4 Gy) IMRT for localized prostate cancer: toxicity and biochemical outcomes. Int J Radiat Oncol Biol Phys 2008, 71(2):330-337.

17. Bolla M, Collette L, Blank L, Warde P, Dubois JB, Mirimanoff RO, Storme G, Bernier J, Kuten A, Sternberg C, Mattelaer J, Lopez Torecilla J, Pfeffer JR, Lino Cutajar C, Zurlo A, Pierart M: Long-term results with immediate androgen suppression and external irradiation in patients with locally advanced prostate cancer (an EORTC study): a phase III randomised trial. Lancet 2002, 360(9327):103-106.

18. D'Amico AV, Chen MH, Renshaw AA, Loffredo M, Kantoff PW: Androgen suppression and radiation vs radiation alone for prostate cancer: a randomized trial. JAMA 2008, 299(3):289-295.

19. Roach M 3rd, Lu J, Pilepichm V, Asbell SO, Mohiuddin M, Terry R, Grignon D, Lawton C, Shipley W, Cox J: Predicting long-term survival, and the need for hormonal therapy: a meta-analysis of RTOG prostate cancer trials. Int J Radiat Oncol Biol Phys 2000, 47(3):617-627.

20. Sharma NK, Li T, Chen DY, Pollack A, Horwitz EM, Buyyounouski MK: Intensity-modulated radiotherapy reduces gastrointestinal toxicity in patients treated with androgen deprivation therapy for prostate cancer. Int J Radiat Oncol Biol Phys 2011, 80(2):437-444.

21. Smeenk RJ, Louwe RJ, Langen KM, Shah AP, Kupelian PA, van Lin EN, Kaanders $\mathrm{JH}$ : An endorectal balloon reduces intrafraction prostate motion during radiotherapy. Int J Radiat Oncol Biol Phys 2012, 83(2):661-669.

22. Peeters ST, Heemsbergen WD, van Putten WL, Slot A, Tabak H, Mens JW, Lebesque JV, Koper PC: Acute and late complications after radiotherapy for prostate cancer: results of a multicenter randomized trial comparing 68 Gy to 78 Gy. Int J Radiat Oncol Biol Phys 2005, 61(4):1019-1034.

23. Bria E, Cuppone F, Giannarelli D, Milella M, Ruggeri EM, Sperduti I, Pinnaro P, Terzoli E, Cognetti F, Carlini P: Does hormone treatment added to radiotherapy improve outcome in locally advanced prostate cancer? meta-analysis of randomized trials. Cancer 2009, 115(15):3446-3456.

24. Jani $A B$, Gratzle J: Late radiotherapy toxicity after prostate cancer treatment: influence of hormonal therapy. Urology 2005, 66(3):566-570. 
25. Zelefsky ML, Fuks Z, Hunt M, Yamada Y, Marion C, Ling CC, Amols H, Venkatraman ES, Leibel SA: High-dose intensity modulated radiation therapy for prostatic cancer: early toxicity and biochemical outcome in 772 patients. Int J Radiat Oncol Biol Phys 2002, 53(5):1111-1116.

26. van der Wielen GJ, van Putten WL, Incrocci L: Sexual function after threedimensional conformal radiotherapy for prostate cancer: results from a dose-escalation trial. Int J Radiat Oncol Biol Phys 2007, 68(2):479-484.

27. Chen CT, Valicenti RK, Lu J, Derose T, Dicker AP, Strup SE, Mulholland SG, Hirsch IH, McGinnis DE, Gomella LG: Does hormonal therapy influence sexual function in men receiving $3 D$ conformal radiation therapy for prostate cancer? Int I Radiat Oncol Biol Phys 2001, 50(3):591-595.

28. Namiki S, Ishidoya S, Ito A, Tochigi T, Numata I, Narazaki K, Yamada S, Takai Y, Arai Y: Five-year follow-up of health-related quality of life after intensitymodulated radiation therapy for prostate cancer. Jpn J Clin Oncol 2009, 39(11):732-738.

doi:10.1186/1748-717X-7-105

Cite this article as: Takeda et al:: Treatment outcome of high-dose image-guided intensity-modulated radiotherapy using intra-prostate fiducial markers for localized prostate cancer at a single institute in Japan. Radiation Oncology 2012 7:105.

\section{Submit your next manuscript to BioMed Central and take full advantage of:}

- Convenient online submission

- Thorough peer review

- No space constraints or color figure charges

- Immediate publication on acceptance

- Inclusion in PubMed, CAS, Scopus and Google Scholar

- Research which is freely available for redistribution 